\title{
Strong electroweak phase transitions without collider traces
}

\author{
A. Ashoorioon* \\ Michigan Center for Theoretical Physics, \\ University of Michigan, Ann Arbor, Michigan 48109-1040, USA \\ T. Konstandint \\ Institut de Física d'Altes Energies, Universitat Autònoma \\ de Barcelona, 08193 Bellaterra, Barcelona, Spain
}

(Dated: October 22, 2018)

\begin{abstract}
We discuss the question if the upcoming generation of collider and low-energy experiments can successfully probe the nature of the electroweak phase transition. In particular, we are interested in phase transitions strong enough for electroweak baryogenesis or even for a production of gravitational radiation observable by the Big Bang Observer.

As an explicit example, we present an analysis in a singlet extension of the Standard Model. We focus on the region in parameter space where the model develops no significant deviation in its low energy phenomenology from the Standard Model. Nevertheless, this class of models can develop a very strong phase transition.
\end{abstract}

\section{INTRODUCTION}

Main objective of the upcoming generation of collider experiments, first and foremost the LHC, is to unravel the nature of electroweak symmetry breaking. The most simplistic model that up to now fits all experimental data is hereby the Standard Model (SM) of particle physics that incorporates the most simple (and perturbatively controllable) scenario: A Higgs sector with a single $S U(2)_{L}$ doublet that breaks the corresponding gauge invariance by means of a vacuum expectation value (VEV).

However, the 'discovery' of this simple scenario would lead to some disappointments:

*Electronic address: amjad@umich.edu

$\dagger$ Electronic address: konstand@ifae.es 
First, the hierarchy problem of the SM suggests that the actual symmetry breaking mechanisms is more complicated and might incorporate supersymmetry, composite Higgs models, Little Higgs models, extra-dimensions, and so on (for a recent review on alternative approaches to electroweak symmetry breaking see ref. [1]). Secondly, a SM Higgs sector lacks a strong electroweak phase transition that is required e.g. for electroweak baryogenesis [2].

In the present work, we discuss the question if upcoming collider and low-energy energy probes compatible with the SM necessary imply the occurrence of a cross-over rather than a strong first-order phase transition. To be specific, we discuss a singlet extension of the SM and show that an electroweak phase transition strong enough for electroweak baryogenesis or even sizable gravitational wave production can in certain models be reconciled with SM collider phenomenology. In particular, we provide a concrete numerical example for this case.

The paper is organized as follows: In sec. II we present the model and set up notation. We also introduce a parametrization of the scalar potential that is more appropriate for the discussion of the phase transition. In sec. III] we discuss possible collider signals and the constraints on the parameter space to avoid a distinction from the SM. We also comment on the possibility of the singlet being a viable dark matter candidate. In secs. IV and V we discuss the electroweak phase transition in the model and give an explicit numerical example. We finally conclude in sec. VI.

\section{THE MODEL}

The model we study in the present work is the minimal singlet extension of the Standard Model as discussed in refs. [3, 4, 5, 6, 7, 8, 9] and its recent revival in form of hidden sector theories [10, 11, 12]. The associated tree-level scalar potential reads ${ }^{1}$

$$
V=V_{S M}+V_{H S}+V_{S}
$$

\footnotetext{
${ }^{1}$ We mostly adhere to the notation of ref. [8] that partially parallels our analysis.
} 
with

$$
\begin{aligned}
V_{S M} & =-\mu^{2} H^{\dagger} H+\lambda_{0}\left(H^{\dagger} H\right)^{2}, \\
V_{H S} & =\frac{a_{1}}{2} H^{\dagger} H S+\frac{a_{2}}{2} H^{\dagger} H S^{2}, \\
V_{S} & =b_{1} S+\frac{b_{2}}{2} S^{2}+\frac{b_{3}}{3} S^{3}+\frac{b_{4}}{4} S^{4},
\end{aligned}
$$

where $H$ denotes the SM $S U(2)_{L}$ scalar Higgs doublet and $S$ denotes the additional scalar singlet. We could shift the singlet field in order to remove the linear contribution from the potential, but it will prove useful to keep it in the following for the regularization of the potential.

In order to discuss the symmetry breaking pattern, we rewrite the fields in terms of vacuum expectation values (VEVs) and fluctuations, using for the neutral Higgs component $H=(v+h) / \sqrt{2}$ and for the singlet field $S=x+s$, leading to

$$
V=-\frac{\mu^{2}}{2} v^{2}+\frac{\lambda_{0}}{4} v^{4}+\frac{a_{1}}{4} v^{2} x+\frac{a_{2}}{4} v^{2} x^{2}+b_{1} x+\frac{b_{2}}{2} x^{2}+\frac{b_{3}}{3} x^{3}+\frac{b_{4}}{3} x^{4} .
$$

In the case $a_{1}=b_{1}=b_{3}=0$, the potential has a $\mathbb{Z}_{2}$ symmetry and the singlet is stable as long this symmetry is not spontaneously broken. However, these conditions are not necessary as we will see in a later section.

The aim of the present paper is to focus on regions in parameter space in which the model develops a strong first-order electroweak phase transition. It turns out that the tree-level potential is in this context better characterized by the following eight conditions that can by traded for the eight parameters in eq. (5), namely the definition of the two VEVs of the broken phase $\bar{v}$ and $\bar{x}$

$$
0=\left.\frac{\partial V}{\partial v}\right|_{v=\bar{v}, x=\bar{x}}, \quad 0=\left.\frac{\partial V}{\partial x}\right|_{v=\bar{v}, x=\bar{x}},
$$

the tree-level Higgs and singlet masses and mixing

$$
\bar{\mu}_{h}^{2}=\left.\frac{\partial^{2} V}{\partial v^{2}}\right|_{v=\bar{v}, x=\bar{x}}, \quad \bar{\mu}_{s}^{2}=\left.\frac{\partial^{2} V}{\partial x^{2}}\right|_{v=\bar{v}, x=\bar{x}}, \quad \bar{\mu}_{h s}^{2}=\left.\frac{\partial^{2} V}{\partial v \partial x}\right|_{v=\bar{v}, x=\bar{x}},
$$

the singlet VEV of the symmetric phase (which by convention we choose to vanish) and the masses of the Higgs and singlet in the symmetric phase

$$
0=\left.\frac{\partial V}{\partial x}\right|_{v=0, x=0}, \quad \mu_{h}^{2}=\left.\frac{\partial^{2} V}{\partial v^{2}}\right|_{v=0, x=0}, \quad \mu_{s}^{2}=\left.\frac{\partial^{2} V}{\partial x^{2}}\right|_{v=0, x=0} .
$$


Notice that the derivative $\partial V /\left.\partial v\right|_{v=0, x=0}$ and $\partial^{2} V /\left.\partial v \partial x\right|_{v=0, x=0}$ automatically vanish such that by these eight parameters the potential is up to second derivatives completely constrained in the broken and symmetric phase.

To replace the eight parameters in eq. (5) by these constraints is always possible, as long as the singlet $\mathrm{VEV} \bar{x}$ does not vanish. In this limit, some of the original parameters could approach infinity as can been seen by their explicit form as given in the appendix. In the examples we make sure that the parameters are small enough to treat the model perturbatively. We also check the correlation between the parameters we use here and the parameters in eq. (5) to ensure that no tuning is embodied by our choice of parameters in the specific numerical examples.

The advantages of this kind of parametrization are apparent: First, with the VEVs and the masses and mixing, the potential is described by physical quantities that will enter in the phenomenological discussion. Second, the second derivatives of the potential in the symmetric phase, $\mu_{h}^{2}$ and $\mu_{s}^{2}$ will lead to two local minima in the potential if chosen positive. Hence, the symmetric phase is even at zero temperature separated from the broken phase by a potential barrier. This is essential for a very strong phase transition.

Even though this model can develop a barrier between the two local minima already at tree-level, one-loop contributions to the potential (and the free energy) are essential to discuss the temperature dependence and we hence include the Coleman-Weinberg contributions [13] for consistency, that read

$$
\begin{aligned}
V_{1} & =\sum_{k} n_{k} G\left[m_{k}^{2}\right]+V_{\text {counterterms }} \\
G(y) & =\frac{y^{2}}{64 \pi^{2}}\left[\ln \left(\frac{y}{Q^{2}}\right)-\frac{3}{2}\right] .
\end{aligned}
$$

Usually the counterterms are arranged as to enforce the conditions in eq. (66). For example, in supersymmetric theories, the soft mass terms can be shifted accordingly which subsequently results in the modification of the physical Higgs mass. However, the present model is not constrained by supersymmetry and we can enforce all constraints eqs. (6)-(8) in our renormalization prescription (see appendix). This has several advantages: First, the physical masses of the Higgs and singlet coincide on tree-level and one-loop level (also the Goldstone bosons remain massless which leads to a technical difficulty that is discussed in ref. [14]). Secondly, the relevant features of the potential to discuss the phase transition are unchanged 
by the one-loop contributions. Last, in case of a heavy singlet, the influence of the singlet on the $v$-dependence of the potential close to the broken minimum automatically ceases and the singlet 'decouples'.

At finite temperature, the effective potential receives in addition the following one-loop contributions

$$
V_{1}^{T \neq 0}=\frac{T^{4}}{2 \pi^{2}} \sum_{k} n_{k} J_{B / F}\left[\left(m_{k}^{2}+\Pi_{k}\right) / T^{2}\right],
$$

with

$$
J_{B / F}(y)=\int_{0}^{\infty} d x x^{2} \ln \left[1 \mp \exp \left(-\sqrt{x^{2}+y}\right)\right],
$$

and the thermal self-energies $\Pi_{k}$. Notice that expanding the expression in the thermalenergies gives rise to the more common one-loop expression without the self-energies and the Daisy-diagrams as detailed in ref. [14]. The thermal self-energies are given in the appendix.

\section{LOW ENERGY PROBES OF THE MODEL}

In this section we discuss several low-energy probes of the model. First, consider the electroweak precision observables (EWPO) that also have been studied in the model at hand in ref. [8, 9]. In leading order, most deviations from the SM values result from the mixing between the Higgs and the singlet. With above given mass terms and the mass eigenstates

$$
h_{1}=\sin \theta s+\cos \theta h, \quad h_{2}=\cos \theta s-\sin \theta h,
$$

the mixing parameter is given by

$$
\tan \theta=\frac{y}{1+\sqrt{1+y^{2}}}, \quad y=\frac{\bar{\mu}_{h s}^{2}}{\bar{\mu}_{h}^{2}-\bar{\mu}_{s}^{2}} .
$$

Then, the deviation of the $T$ parameter from its SM value is in leading order e.g. given by

$$
\begin{aligned}
T-T_{S M}= & \left(\frac{3}{16 \pi \sin \theta_{W}^{2}}\right)\left\{\frac{m_{Z}^{2}}{m_{W}^{2}}\left(\frac{m_{h}^{2}}{m_{h}^{2}-m_{Z}^{2}}\right) \log \frac{m_{h}^{2}}{m_{Z}^{2}}-\left(\frac{m_{h}^{2}}{m_{h}^{2}-m_{W}^{2}}\right) \log \frac{m_{h}^{2}}{m_{W}^{2}}\right. \\
& -\cos ^{2} \theta\left[\frac{m_{Z}^{2}}{m_{W}^{2}}\left(\frac{m_{1}^{2}}{m_{1}^{2}-m_{Z}^{2}}\right) \log \frac{m_{1}^{2}}{m_{Z}^{2}}-\left(\frac{m_{1}^{2}}{m_{1}^{2}-m_{W}^{2}}\right) \log \frac{m_{1}^{2}}{m_{W}^{2}}\right] \\
& \left.-\sin ^{2} \theta\left[\frac{m_{Z}^{2}}{m_{W}^{2}}\left(\frac{m_{2}^{2}}{m_{2}^{2}-m_{Z}^{2}}\right) \log \frac{m_{2}^{2}}{m_{Z}^{2}}-\left(\frac{m_{2}^{2}}{m_{2}^{2}-m_{W}^{2}}\right) \log \frac{m_{2}^{2}}{m_{W}^{2}}\right]\right\} .
\end{aligned}
$$

Hence, if mixing between the Higgs and the singlet is suppressed, the singlet extension cannot be distinguished from the SM with Higgs mass $m_{h}=m_{1}$. So without further ado we use the freedom in the potential to set the mixing to zero, $\bar{\mu}_{h s}^{2}=0$. 

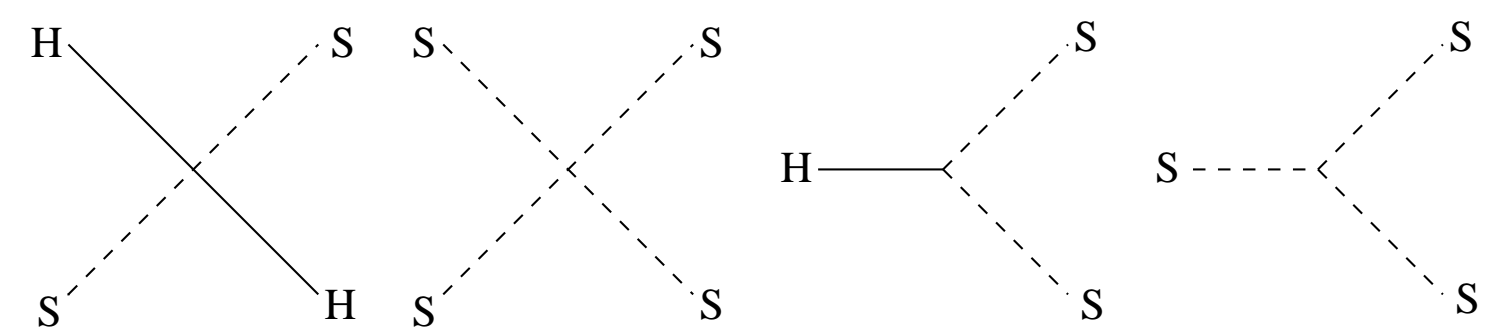

FIG. 1: The additional Feynman rules involving the singlets under the assumption of vanishing Higgs-singlet mixing, $\bar{\mu}_{h s}^{2}=0$.

Next, we consider the detection of the additional degree of freedom in collider experiments. In order to do so, consider the Feynman rules arising from the tree-level potential. In particular, we assume that there is no mixing between the Higgs and the singlet, $\bar{\mu}_{h s}^{2}=0$, to reduce the constraints on the EWPO as explained before.

For the Higgs self-interactions one finds at tree-level

$$
\left.\frac{\partial^{3} V}{\partial v^{3}}\right|_{v=\bar{v}, x=\bar{x}}=\frac{3 \bar{\mu}_{h}^{2}}{\bar{v}},\left.\quad \frac{\partial^{4} V}{\partial v^{4}}\right|_{v=\bar{v}, x=\bar{x}}=\frac{3 \bar{\mu}_{h}^{2}}{\bar{v}^{2}},
$$

and hence exactly the SM relations. There will be a small modification due to the additional one-loop contribution of the singlet, but this effect is too small to be measured in the near future. Notice that this is somewhat in contradiction with the claim in ref. [15] that a strong phase transition necessarily leaves traces in the Higgs self-couplings (see also ref. [12] for the corresponding discussion with many strongly coupled singlets and refs. [16, 17] for an analysis in a two Higgs doublet model).

Compared to the SM there are new interaction terms with the singlet which for vanishing mixing, $\bar{\mu}_{h s}=0$, read

$$
\begin{aligned}
\left.\frac{\partial^{3} V}{\partial x^{2} \partial v}\right|_{v=\bar{v}, x=\bar{x}} & =\frac{\left(2 \mu_{h}^{2}+\bar{\mu}_{h}^{2}\right) \bar{v}}{\bar{x}^{2}} \\
\left.\frac{\partial^{3} V}{\partial x^{3}}\right|_{v=\bar{v}, x=\bar{x}} & =\frac{\left(2 \mu_{s}^{2}+4 \bar{\mu}_{s}^{2}\right) \bar{x}^{2}-\left(4 \mu_{h}^{2}+2 \bar{\mu}_{h}^{2}\right) \bar{v}^{2}}{\bar{x}^{3}} \\
\left.\frac{\partial^{4} V}{\partial x^{2} \partial v^{2}}\right|_{v=\bar{v}, x=\bar{x}} & =\frac{2 \mu_{h}^{2}+\bar{\mu}_{h}^{2}}{\bar{x}^{2}}, \\
\left.\frac{\partial^{4} V}{\partial x^{4}}\right|_{v=\bar{v}, x=\bar{x}} & =\frac{\left(6 \mu_{s}^{2}+6 \bar{\mu}_{s}^{2}\right) \bar{x}^{2}-\left(6 \mu_{h}^{2}+3 \bar{\mu}_{h}^{2}\right) \bar{v}^{2}}{\bar{x}^{4}},
\end{aligned}
$$

leading to Feynman rules depicted in Fig. 1, Notice that only the cubic self-interaction of the singlet contains an odd number of singlets such that the singlet cannot decay on tree-level. 
If the singlet is much lighter than the Higgs particle, $2 m_{s}<m_{h}$, the Higgs can decay into singlets. In this case the Higgs might even become invisible at colliders, if the singlets have only a small decay rate into SM particles [18, 19]. In the following, we assume the singlet to be heavier than this bound. Typically, the Higgs could also decay into two virtual singlets and then lead to quite characteristic decays into four SM particles. However, this is only possible if the singlet mixes with the Higgs thus opening the singlet decay channels into SM particles (see [9] for a detailed discussion of this case). Since small mixing also implies a rather long lifetime for the singlet (see below), the most promising channel is the production of two singlets by a Higgs with resulting missing energy signal. If the singlets are rather heavy this requires a Higgs that is far off-shell what makes this channel unfeasible at LHC.

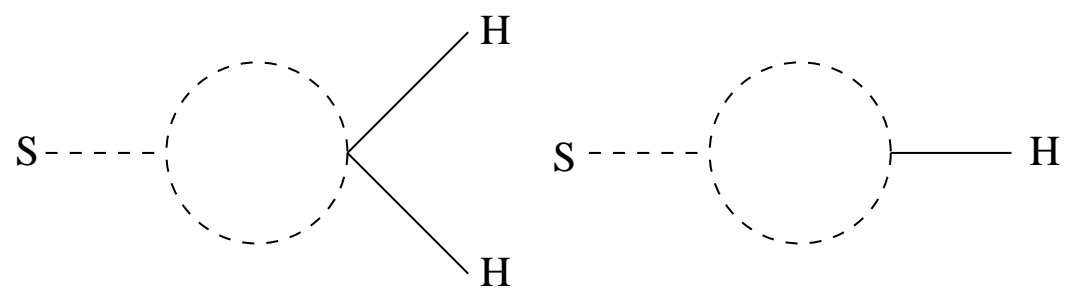

FIG. 2: One-loop diagrams that contribute to decay and mixing of the singlet with the Higgs.

Before the discussion of the phase transition, we comment on the possibility of the singlet being stable and hence a viable dark matter candidate. In fact, the decay is automatically suppressed if the mixing between Higgs and singlet is. This can be seen as follows. A similar diagram to the decay diagram arises as a one-loop contribution to the singlet-Higgs mixing (both depicted in Fig. 22). Both diagrams are in fact divergent and have to be regularized simultaneously by adjusting the mixing parameter $\bar{\mu}_{h s}^{2}$. This is possible since the vertex $h s$ and the vertex $h h s$ are on tree-level as well as on one-loop proportional to each other (with a relative factor $\bar{v}$ in both cases). On tree-level, both vertices are in addition proportional to $\bar{\mu}_{h s}$. This in turn implies that if the parameters are arranged to suppress mixing, singlet decay will also be suppressed at least by two loop orders and by the corresponding Higgssinglet couplings.

If the decay rate of the singlet vanished (or at least were smaller than today's Hubble parameter), the annihilation rate would determine today's singlet abundance. The annihilation cross section resulting from the vertices hhss and hss are of electroweak scale which makes the singlet a good WIMP candidate, see Fig. 3, Due to the WIMP miracle, the 


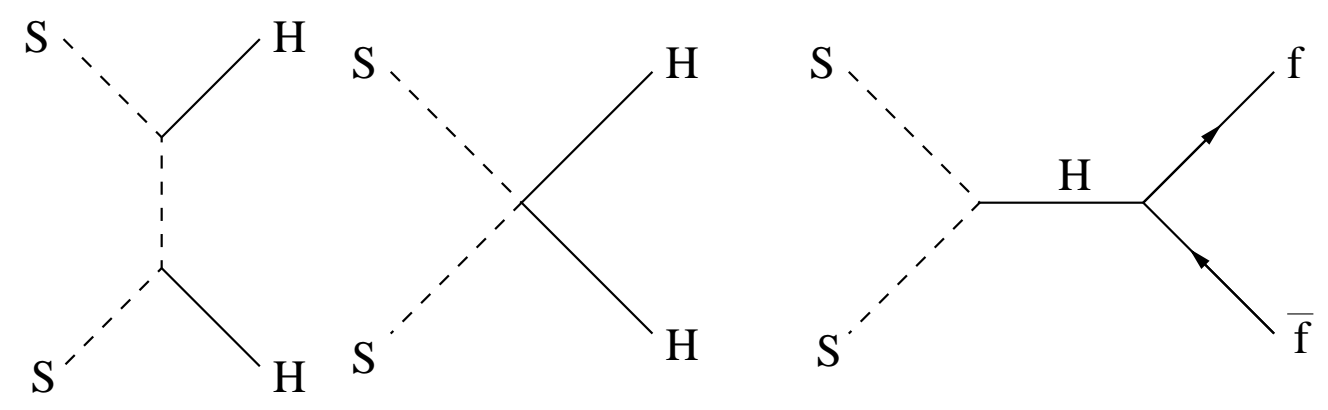

FIG. 3: Dominant contributions to singlet annihilation.

resulting singlet abundance today would then be automatically in the right range to explain the observed dark matter density [20]. However, in the present model, the singlet is only protected from decaying if a (unbroken) $\mathbb{Z}_{2}$ symmetry is imposed which is not the case we are interested in. Hence, generically the singlet is not a viable dark matter candidate.

Finally, we comment on the realization of baryogenesis in the present model in case the electroweak phase transition is strongly first-order. Notice that the current framework does so far not contain a source of $\mathrm{CP}$ violation that is sufficient for viable electroweak baryogenesis. The simplest way of introducing CP violation is by resorting to dimension-six operators, as done in ref. [21]. Dimension-six operators that couple the top to the Higgs field can provide sufficient $\mathrm{CP}$ violation without changing collider phenomenology but give rise to electric dipole moments in reach of the next generation of experiments [22]. Even though this seems possible, we would like to point out that the main intent of the present work is not to provide an example for electroweak baryogenesis with Standard Model collider phenomenology. The aim is rather to demonstrate that collider experiments are in general not able to determine the strength of the phase transition. For example, a strong phase transition in the MSSM requires some tuning in the Higgs and stop sectors such that a similar construction as presented here also could relax these tunings without collider traces beyond the generic MSSM (for specific collider traces of the MSSM with a light right-handed stop see e.g. ref. [23]). In this case the chargino [24, 25, 26] or neutralino [27] sector provides the required source of $\mathrm{CP}$ violation.

In conclusion, the singlet model is indistinguishable from the SM by the upcoming collider experiments as long as the mixing between the singlet and the Higgs is small and the singlet is heavy enough to prohibit the Higgs to decay into singlet pairs. 


\section{THE PHASE TRANSITION AT TREE LEVEL}

In this section, main characteristics of the electroweak phase transition are discussed. Most aspects of the phase transition can be discussed while focusing on the tree-level and inclusion of one-loop contributions (at zero temperature) lead only to minor deviations due to the regularization scheme used. Motivated by the analysis in the last section, we only consider the case without mixing between the singlet and the Higgs in the broken phase, $\bar{\mu}_{h s}^{2}=0$.

Current measurements of the electroweak precision observables favor a rather light Higgs (at least in the case of the $\mathrm{SM}$ ), such that reasonable values for the Higgs mass lie in the range $\bar{\mu}_{h}^{2}=(114-160 \mathrm{GeV})^{2}[28]$. To avoid the detection of the singlet in up-coming collider experiments, the singlet should be heavy enough to prohibit Higgs decay into singlet pairs, $4 \bar{\mu}_{s}^{2} \gtrsim \bar{\mu}_{h}^{2}$. Besides it should be large enough to lead to a sizable potential difference between the broken and the symmetric phase (to lead to a sizable phase transition temperature) that at tree-level is given by

$$
\Delta V=\frac{1}{12}\left(\left(\mu_{h}^{2}-\bar{\mu}_{h}^{2}\right) \bar{v}^{2}+\left(\mu_{s}^{2}-\bar{\mu}_{s}^{2}\right) \bar{x}^{2}\right),
$$

what can be easily arranged by choosing the singlet mass in the broken and symmetric phase suitably.

The singlet VEV in the broken phase, $\bar{x}$, constitutes an additional free parameter. Its value is bounded from above, since a too large path between the symmetric and the broken phase increases the tunnel action substantially and can prohibit tunneling altogether. On the other hand, too small values of $\bar{x}$ lead in conjuncture with sizable masses to very large values of the quartic coupling $b_{4}$ which would spoil the validity of perturbative approaches. Typically, the singlet VEV is hence confined to a range $\bar{x} \sim 150-250 \mathrm{GeV}$.

In the present model, the strength of the phase transition is mostly given by the treelevel parameters of the potential. This is in contrast to e.g. the SM, where a potential barrier between the broken and symmetric phase is only generated by thermal one-loop contributions [29], or to the singlet extensions without singlet VEVs, where the barrier is generated by Coleman-Weinberg terms [11]. Hence, a phase transition strong enough to avoid sphaleron washout [2], $v(T) / T>1$, is easily achieved by adjusting the tree-level parameters accordingly and is a generic feature of the model. This is thoroughly demonstrated 
in ref. [8] and can also be seen from the analysis in the nMSSM that contains a similar scalar sector [30, 31]. In the following we will focus on the more exceptional case of a phase transition that is strong enough to even give rise to substantial gravitational radiation.

In order to analyze a first-order phase transition, we determine the three-dimensional, Euclidean tunnel action of the bounce solution of the scalar fields, $v(\rho)$ and $x(\rho)$,

$$
S_{3}=4 \pi \int d \rho \rho^{2}\left[\frac{1}{2}\left(\frac{d v}{d \rho}\right)^{2}+\frac{1}{2}\left(\frac{d x}{d \rho}\right)^{2}+V(v, x, T)\right] .
$$

The tunneling usually proceeds for temperatures with $S_{3} / T \approx 140$ (for generalities of semiclassical tunneling see refs. [32, 33, 34]; the concrete formulas we use in the present analysis are taken from ref. [30]). Subsequently we determine the characteristics of the phase transition that are relevant for electroweak baryogenesis and the production of gravitational radiation. These include the $\mathrm{VEV}$ to temperature ratio right after the phase transition, $v / T$, that controls the suppression of sphaleron processes, the latent heat normalized to the radiation energy of the plasma, $\alpha$, and the inverse duration of the phase transition in units of the Hubble parameter, $\beta / H$, that in terms of the tunnel action are given by

$$
\begin{gathered}
\alpha=\frac{30 \epsilon}{\pi^{2} g_{*} T^{4}}, \quad \frac{\beta}{H}=T \frac{d}{d T} \frac{S_{3}}{T} \\
\epsilon=V(v, x, T)-V(0,0, T)-T \frac{d}{d T}(V(v, x, T)-V(0,0, T)) .
\end{gathered}
$$

The tunnel action $S_{3}$ diverges at a temperature $T_{c}$ when the local minima of the broken and symmetric phase equal in potential and is a decreasing function in temperature. To achieve a rather strong phase transition, it is necessary to have already at zero temperature a potential barrier between the local minima, because otherwise the phase transition proceeds at rather high energies, $T \approx T_{c}$, and rather fast, $\beta / H \gg 100$. This potential barrier can be achieved by choosing the corresponding parameters of the potential in the symmetric phase positive

$$
\mu_{s}^{2}, \mu_{h}^{2}>0
$$

On the other hand, if $\mu_{h}^{2}$ is chosen rather large, the tunnel probability is too small for the phase transition to happen, $S_{3} / T \gtrsim 140$, and the model is stuck in the symmetric phase and phenomenologically not viable. Accordingly, the Higgs mass in the symmetric phase, $\mu_{h}^{2}$, has to be rather small for the phase transition to happen. For one typical class of models the phase transition proceeds as follows: At very high temperatures, the effective potential 
has only one local minimum which is situated at vanishing Higgs VEV and at a singlet VEV that ranges somewhere between 0 and $\bar{x}$. At some temperature related to the singlet mass, the potential develops two minima $x_{1 / 2}$ and the minimum closer to the origin $x_{1}$ is the lower of both in potential. At even lower temperature, the minimum at $x_{2}$ becomes unstable and another local minimum with non vanishing Higgs VEV develops. As soon as this new local minimum is significantly below the one close to the origin, tunneling might happen. This situation is depicted in Fig. 4. In order to tunnel, the scalar fields have to overcome a ridge between the two valleys, thus leading to a substantial tunnel action. A detailed numerical example including the one-loop corrections and a numerical evaluation of the tunnel action is given in the next section.
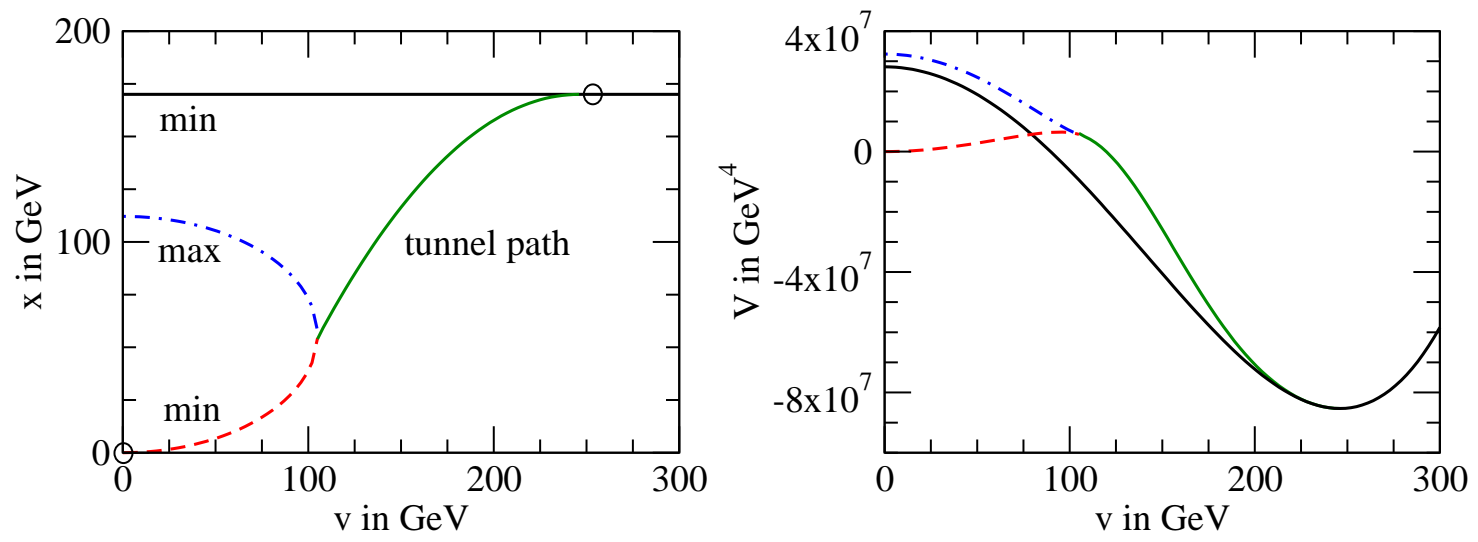

FIG. 4: An example of the paths of the extrema as functions $x(v)$ in scalar field space and the corresponding values of the potential. The two circles denote the local minima in the potential. For small Higgs VEV $v$, the potential has two minima and one maximum with respect to $x$. For large $v$ only one minimum remains. The fields have to cross the ridge to tunnel from the symmetric to the broken phase. In addition, the plot shows a typical path we choose to determine the tunneling action.

\section{A NUMERICAL EXAMPLE}

In this section, we present the analysis of the specific numerical example for a rather strong phase transition without traces in upcoming collider experiments. The analysis uses the one-loop free energy including thermal masses (to account for Daisy diagrams). The 
concrete values we use are

$$
\begin{gathered}
\mu_{h}^{2}=(20 \mathrm{GeV})^{2}, \quad \mu_{s}^{2}=(150 \mathrm{GeV})^{2}, \quad \bar{x}=170 \mathrm{GeV} \\
\bar{\mu}_{s}^{2} \approx(190 \mathrm{GeV})^{2}, \quad \bar{\mu}_{h s}^{2}=(0 \mathrm{GeV})^{2}, \quad \bar{\mu}_{h}^{2}=(120 \mathrm{GeV})^{2} .
\end{gathered}
$$

There are in principle several possibilities to determine the tunnel action. Since, there are only two scalar fields present, one could try to find the bounce solution of the tunnel action by combining the methods of scanning and over/under-shooting [35]. However, in the present case the convergence of this algorithm is very poor, since close to the symmetric phase the scalar fields have to follow the ridge of the potential with high accuracy. The most sophisticated method would be to use one of the algorithms designed to find bounce solutions in the case of several scalar fields [36, 37]. However, these methods are rather involved and an estimate of the tunnel action is sufficient for the following discussion. We use the following method: First we choose a path by hand and then determine the corresponding bounce solution by over/under-shooting. The bounce solution constitutes a saddle point of the action with only one negative eigenvalue in the functional determinant [38]. This negative eigenvalue is eliminated by the over/under-shooting method while the bounce solution is a minimum of the action with respect to changing the path in scalar field space. One path with nearly minimal action is the one that follows the ridge near the symmetric phase closely until its end and then smoothly connects with the broken phase. Since the mass of the singlet is in the present example significantly larger than the Higgs mass, the tunnel action also prefers a path that leaves the broken phase in the direction of the Higgs VEV. This situation is depicted in Fig. 4 .

In Fig. 5, the tunnel action is depicted as a function of temperature for several values of $\bar{\mu}_{s}^{2}$ and the remaining parameters as in eq. (25). By adjusting $\bar{\mu}_{s}^{2}$, a model can be found in which the scalar fields barely tunnel. The phase transition proceeds in this case very slowly and with only a few bubbles per Hubble volume and a rather large latent heat results. The corresponding characteristics of the phase transition are given in table $\mathbb{1}$.

In all cases the phase transition is strong enough for viable baryogenesis, $v / T \gtrsim 1$. Finally we would like to comment on gravitational wave production during the phase transition. For a general discussion of the different arising contributions to gravitational wave production we refer the reader to refs. [30, 39, 40]. For gravitational wave production observable by the the planned Big Bang Observer [41], a phase transition at electroweak scales typically 


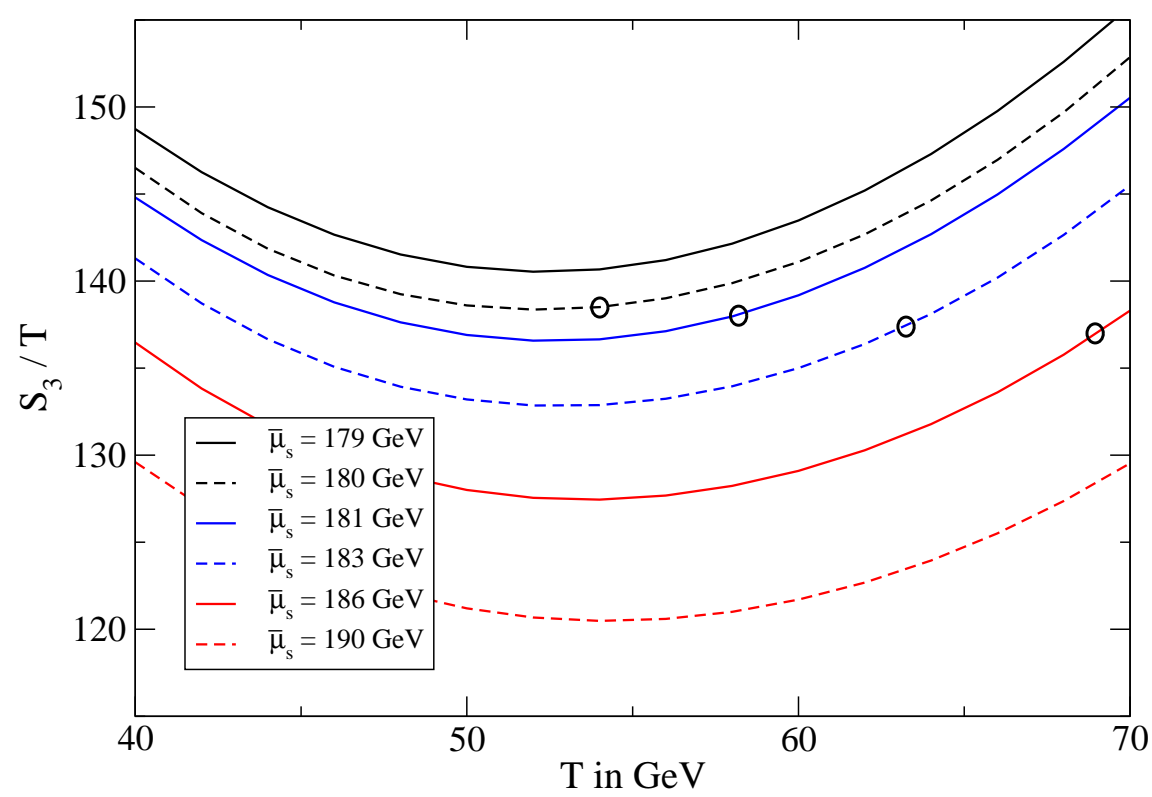

FIG. 5: The tunnel action $S_{3} / T$ as a function of the temperature for different values of $\bar{\mu}_{s}$. The circles denote the temperature at the end of the phase transition determined with the methods from ref. [30].

\begin{tabular}{|c||c|c|c|c|}
\hline $\bar{\mu}_{s} / \mathrm{GeV}$ & $\alpha$ & $\beta / H$ & $v / T$ & $T / \mathrm{GeV}$ \\
\hline \hline 190 & 0.14 & 121 & 3.1 & 75 \\
\hline 186 & 0.18 & 88 & 3.4 & 69 \\
\hline 183 & 0.25 & 53 & 3.7 & 63 \\
\hline 181 & 0.33 & 25 & 4.0 & 57 \\
\hline 180 & 0.42 & 8 & 4.2 & 54 \\
\hline 179 & \multicolumn{5}{|c}{ symmetric phase stable } \\
\hline
\end{tabular}

TABLE I: Sets of parameters corresponding to Fig. 5 .

requires $\alpha \gtrsim 0.1$ and $\beta / H \lesssim 200$ for a signal from bubble collisions [42, 43] and a slightly stronger phase transition for a signal from turbulence [44, 45]. The gravitational wave spectra resulting from colliding bubbles as given in ref. [43] and from turbulence as given in refs. [30, 45] and for the parameters in Tab. 1] are shown in Fig. 6.

We conclude that a phase transition strong enough for electroweak baryogenesis is quite generic in the present model. Additionally, with a tuning in the parameter $\bar{\mu}_{s}$ on the few 


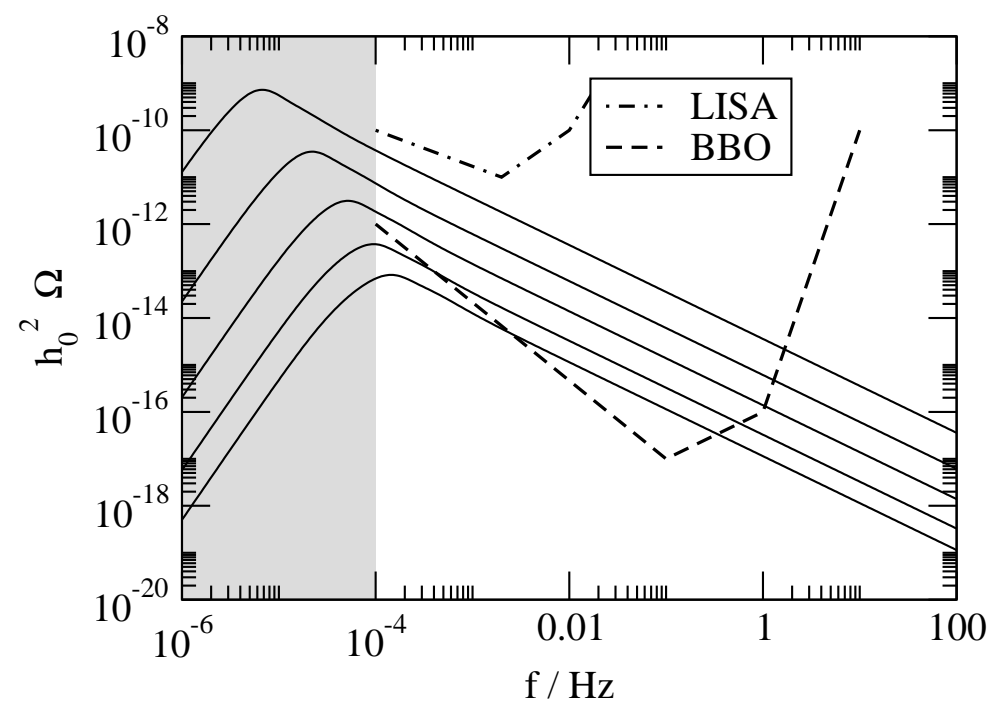

FIG. 6: The spectra of gravitational wave generation for the parameters given in Tab. I (the parameter $\alpha$ is increasing from bottom to top). The dashed lines show the sensitivity of the LISA and $\mathrm{BBO}$ experiments. In the shaded region the sensitivity of both experiments drops significantly.

percent level, a model can be found that even can lead to gravitational wave production observable by the Big Bang Observer.

\section{DISCUSSION}

Main concern of the present work is the question if experimental insights about the Higgs sector from upcoming collider experiments will be sufficient to reliably predict the nature of the electroweak phase transition. In order to do so we discussed a singlet extension of the SM as a showcase. Our main conclusion is that an extremely strong first-order phase transition could have occurred in the early Universe without leading to any collider signals beyond the SM.

Main constraint on the model is that the mixing between the Higgs and the singlet is suppressed. Besides, the singlet has to be heavy enough to avoid Higgs decay into singlet pairs. Interestingly, the singlet is under these assumptions (rather) stable which further reduces the chances of the model to be distinguished from the SM at colliders. On the other hand, vanishing mixing between the Higgs and the singlet is not protected by any symmetry in the present model (and also not stable under radiative corrections) such that mixing is not expected to vanish exactly and should be experimentally testable at some stage. The 
prospects to distinguish the present model from the SM are hereby significantly higher with the next generation of linear collider experiments than with the LHC if mixing is small.

With the tree-level parametrization we used in the present analysis, regions in parameter space with a first-order phase transition are easily found:

- A first-order phase transition strong enough for electroweak baryogenesis, $\phi / T \gtrsim 1$, is a quite generic feature of the present model.

- A first-order phase transition strong enough for a gravitational wave production that is at least observable by the Big Bang Observer typically requires a rather small Higgs mass in the symmetric phase and a tuning of the singlet masses (in the symmetric or broken phases) on the few percent level.

We would like to emphasize that in the present analysis we focused from the beginning on regions in parameter space with no collider signals beyond the SM. Nevertheless, in the general case it is found that regions in parameter space with strong phase transitions can be correlated with specific collider signatures as e.g. exotic final states [8, 9].

Finally, notice that the model discussed here is most probably the minimal model that reconciles SM collider phenomenology with a strong first-order phase transition. The essential ingredient is hereby that the new scalar field has a changing VEV during the electroweak phase transition. If fields are added to the SM that are coupled strongly to the Higgs but do not obtain a VEV, the Higgs self-couplings are typically modified compared to the SM value by the same one-loop contributions to the scalar potential that strengthen the phase transition. This is basically the argument put forward in ref. [15] supporting the claim that a strong phase transition will generically leave significant traces in the interactions of the Higgs sector. The present work provides a concrete counterexample to this claim.

\section{Acknowledgments}

We would like to thank J. R. Espinosa and A. T. Pierce for many helpful discussions. T. K. acknowledges support by the Marie Curie Research \& Training Network 'UniverseNet' (MRTN-CT-2006-035863). A. A. is supported by NSERC of Canada and MCTP. 


\section{APPENDIX A: REPARAMETRIZATION}

In this section, we display the specific reparametrization of the parameters in the potential. In order to use this description also for the determination of the counterterms, we define in the broken phase the first derivatives

$$
\bar{d}_{h}=\left.\frac{\partial V}{\partial v}\right|_{v=\bar{v}, x=\bar{x}}, \quad \bar{d}_{s}=\left.\frac{\partial V}{\partial x}\right|_{v=\bar{v}, x=\bar{x}},
$$

the tree-level Higgs and singlet masses and mixing

$$
\bar{\mu}_{h}^{2}=\left.\frac{\partial^{2} V}{\partial v^{2}}\right|_{v=\bar{v}, x=\bar{x}}, \quad \bar{\mu}_{s}^{2}=\left.\frac{\partial^{2} V}{\partial x^{2}}\right|_{v=\bar{v}, x=\bar{x}}, \quad \bar{\mu}_{h s}^{2}=\left.\frac{\partial^{2} V}{\partial v \partial x}\right|_{v=\bar{v}, x=\bar{x}},
$$

the first derivative in the symmetric phase and the second derivatives of the potential in the symmetric phase

$$
d_{s}=\left.\frac{\partial V}{\partial x}\right|_{v=0, x=0}, \quad \mu_{h}^{2}=\left.\frac{\partial^{2} V}{\partial v^{2}}\right|_{v=0, x=0}, \quad \mu_{s}^{2}=\left.\frac{\partial^{2} V}{\partial x^{2}}\right|_{v=0, x=0} .
$$

The resulting parameters then read

$$
\begin{aligned}
b_{1} & =d_{s}, \quad \mu^{2}=-\mu_{h}^{2}, \quad b_{2}=\mu_{s}^{2}, \quad \lambda_{0}=-\frac{\bar{d}_{h}-\bar{\mu}_{h}^{2} \bar{v}}{2 \bar{v}^{3}}, \\
a_{1} & =-\frac{-6 \bar{d}_{h}+4 \mu_{h}^{2} \bar{v}+2 \bar{\mu}_{h}^{2} \bar{v}+2 \bar{\mu}_{h s}^{2} \bar{x}}{\bar{v} \bar{x}}, \quad a_{2}=\frac{-3 \bar{d}_{h}+2 \mu_{h}^{2} \bar{v}+\bar{\mu}_{h}^{2} \bar{v}+2 \bar{\mu}_{h s}^{2} \bar{x}}{\bar{v} \bar{x}^{2}}, \\
b_{3} & =-\frac{3 \bar{d}_{h} \bar{v}-2 \mu_{h}^{2} \bar{v}^{2}-\bar{\mu}_{h}^{2} \bar{v}^{2}+6 d_{s} \bar{x}-6 \bar{d}_{s} \bar{x}+\mu_{h s}^{2} \bar{v} \bar{x}+4 \mu_{s}^{2} \bar{x}^{2}+2 \bar{\mu}_{s}^{2} \bar{x}^{2}}{2 \bar{x}^{3}}, \\
b_{4} & =-\frac{-3 \bar{d}_{h} \bar{v}+2 \mu_{h}^{2} \bar{v}^{2}+\bar{\mu}_{h}^{2} \bar{v}^{2}-4 d_{s} \bar{x}+4 \bar{d}_{s} \bar{x}-2 \mu_{s}^{2} \bar{x}^{2}-2 \bar{\mu}_{s}^{2} \bar{x}^{2}}{2 \bar{x}^{4}} .
\end{aligned}
$$

From these formulas it is clear that the limit $\bar{x} \rightarrow 0$ leads in certain cases to a divergent set of parameters. The very same relations can be used to determine the counterterms as functions of the derivatives of the one-loop potential.

\section{APPENDIX B: THERMAL MASSES}

The scalar masses are derived from the scalar potential as given in eq. (5)

$$
V=-\frac{\mu^{2}}{2} v^{2}+\frac{\lambda_{0}}{4} v^{4}+\frac{a_{1}}{4} v^{2} x+\frac{a_{2}}{4} v^{2} x^{2}+b_{1} x+\frac{b_{2}}{2} x^{2}+\frac{b_{3}}{3} x^{3}+\frac{b_{4}}{3} x^{4} .
$$

and can be recast using the relations in the previous section. The Higgs/singlet mass matrix reads

$$
m_{H S}^{2}=\left(\begin{array}{cc}
-\mu^{2}+3 \lambda_{0} v^{2}+\frac{1}{2} a_{1} x+\frac{1}{2} a_{2} x^{2} & \frac{1}{2} a_{1} v+a_{2} v x \\
\frac{1}{2} a_{1} v+a_{2} v x & \frac{1}{2} a_{2} v^{2}+b_{2}+2 b_{3} x+3 b_{4} x^{4}
\end{array}\right)
$$


and the Goldstone masses are given by

$$
m_{G}^{2}=-\mu^{2}+3 \lambda_{0} v^{2}+\frac{1}{2} a_{1} x+\frac{1}{2} a_{2} x^{2}
$$

The masses of the gauge bosons are

$$
m_{g b}^{2}=\left(\begin{array}{cccc}
\frac{g^{2}}{4} v^{2} & 0 & 0 & 0 \\
0 & \frac{g^{2}}{4} v^{2} & 0 & 0 \\
0 & 0 & \frac{g^{2}}{4} v^{2} & \frac{g g^{\prime}}{4} v^{2} \\
0 & 0 & \frac{g g^{\prime}}{4} v^{2} & \frac{g^{\prime 2}}{4} v^{2}
\end{array}\right)
$$

and the top quark mass is given by

$$
m_{t}^{2}=\frac{y_{t}^{2}}{2} v^{2}
$$

We take account of the corresponding self-energies at finite temperature in leading order. For the Higgs/singlet bosons they read

$$
\Pi_{H S}=\left(\begin{array}{cc}
\left(\frac{3}{16} g^{2}+\frac{1}{16} g^{\prime 2}+\frac{1}{2} \lambda_{0}+\frac{1}{4} y_{t}^{2}+\frac{1}{24} a_{2}\right) T^{2} & 0 \\
0 & \left(\frac{1}{4} b_{4}+\frac{1}{6} a_{2}\right) T^{2}
\end{array}\right)
$$

and for the Goldstone bosons

$$
\Pi_{G}=\left(\frac{3}{16} g^{2}+\frac{1}{16} g^{\prime 2}+\frac{1}{2} \lambda_{0}+\frac{1}{4} y_{t}^{2}+\frac{1}{24} a_{2}\right) T^{2}
$$

Finally, for the longitudinal gauge bosons one finds

$$
\Pi_{g b}=\left(\begin{array}{cccc}
\frac{11}{6} g^{2} T^{2} & 0 & 0 & 0 \\
0 & \frac{11}{6} g^{2} T^{2} & 0 & 0 \\
0 & 0 & \frac{11}{6} g^{2} T^{2} & 0 \\
0 & 0 & 0 & \frac{11}{6} g^{\prime 2} T^{2}
\end{array}\right) .
$$

[1] C. Grojean, "New Approaches To Electroweak Symmetry Breaking," Phys. Usp. 50 (2007) 1 [Usp. Fiz. Nauk 177 (2007) 3].

[2] G. R. Farrar and M. E. Shaposhnikov, "Baryon Asymmetry Of The Universe In The Standard Electroweak Theory," Phys. Rev. D 50 (1994) 774 [hep-ph/9305275].

[3] J. R. Espinosa and M. Quiros, "The Electroweak phase transition with a singlet," Phys. Lett. B 305 (1993) 98 [arXiv:hep-ph/9301285]. 
[4] J. Choi and R. R. Volkas, "Real Higgs singlet and the electroweak phase transition in the standard model, (UM-P-93/80, OZ-93/20)," Phys. Lett. B 317 (1993) 385 arXiv:hep-ph/9308234.

[5] A. Datta and A. Raychaudhuri, "Next to minimal Higgs: Mass bounds and search prospects," Phys. Rev. D 57 (1998) 2940 arXiv:hep-ph/9708444.

[6] D. O'Connell, M. J. Ramsey-Musolf and M. B. Wise, "Minimal Extension of the Standard Model Scalar Sector," Phys. Rev. D 75 (2007) 037701 arXiv:hep-ph/0611014.

[7] O. Bahat-Treidel, Y. Grossman and Y. Rozen, "Hiding the Higgs at the LHC," JHEP 0705 (2007) 022 arXiv:hep-ph/0611162].

[8] S. Profumo, M. J. Ramsey-Musolf and G. Shaughnessy, "Singlet Higgs Phenomenology and the Electroweak Phase Transition," JHEP 0708 (2007) 010 [arXiv:0705.2425 [hep-ph]].

[9] V. Barger, P. Langacker, M. McCaskey, M. J. Ramsey-Musolf and G. Shaughnessy, "LHC Phenomenology of an Extended Standard Model with a Real Scalar Singlet," Phys. Rev. D 77 (2008) 035005 arXiv:0706.4311 [hep-ph]].

[10] R. Schabinger and J. D. Wells, "A minimal spontaneously broken hidden sector and its impact on Higgs boson physics at the Large Hadron Collider," Phys. Rev. D 72 (2005) 093007 arXiv:hep-ph/0509209].

[11] J. R. Espinosa and M. Quiros, "Novel effects in electroweak breaking from a hidden sector," Phys. Rev. D 76 (2007) 076004 arXiv:hep-ph/0701145].

[12] J. R. Espinosa, T. Konstandin, J. M. No and M. Quiros, "Some Cosmological Implications of Hidden Sectors," Phys. Rev. D 78 (2008) 123528 [arXiv:0809.3215 [hep-ph]].

[13] S. R. Coleman and E. Weinberg, "Radiative Corrections As The Origin Of Spontaneous Symmetry Breaking," Phys. Rev. D 7 (1973) 1888.

[14] C. Delaunay, C. Grojean and J. D. Wells, "Dynamics of Non-renormalizable Electroweak Symmetry Breaking," JHEP 0804 (2008) 029 [arXiv:0711.2511 [hep-ph]].

[15] A. Noble and M. Perelstein, "Higgs Self-Coupling as a Probe of Electroweak Phase Transition," [hep-ph/0711.3018].

[16] S. Kanemura, Y. Okada and E. Senaha, "Electroweak baryogenesis and quantum corrections to the triple Higgs boson coupling," Phys. Lett. B 606 (2005) 361 arXiv:hep-ph/0411354.

[17] M. Aoki, S. Kanemura and O. Seto, "Neutrino mass, Dark Matter and Baryon Asymmetry via TeV-Scale Physics without Fine-Tuning," Phys. Rev. Lett. 102 (2009) 051805 arXiv:0807.0361 [hep-ph]]. 
[18] M. C. Bento, O. Bertolami, R. Rosenfeld and L. Teodoro, "Self-interacting dark matter and invisibly decaying Higgs," Phys. Rev. D 62 (2000) 041302 [arXiv:astro-ph/0003350].

[19] C. P. Burgess, M. Pospelov and T. ter Veldhuis, "The minimal model of nonbaryonic dark matter: A singlet scalar," Nucl. Phys. B 619 (2001) 709 arXiv:hep-ph/0011335.

[20] J. McDonald, "Gauge Singlet Scalars as Cold Dark Matter," Phys. Rev. D 50 (1994) 3637 arXiv:hep-ph/0702143.

[21] D. Bodeker, L. Fromme, S. J. Huber and M. Seniuch, "The baryon asymmetry in the standard model with a low cut-off," JHEP 0502 (2005) 026 arXiv:hep-ph/0412366].

[22] S. J. Huber, M. Pospelov and A. Ritz, "Electric dipole moment constraints on minimal electroweak baryogenesis," Phys. Rev. D 75 (2007) 036006 arXiv:hep-ph/0610003.

[23] A. Menon and D. E. Morrissey, "Higgs Boson Signatures of MSSM Electroweak Baryogenesis," arXiv:0903.3038 [hep-ph].

[24] A. E. Nelson, D. B. Kaplan and A. G. Cohen, "Why there is something rather than nothing: Matter from weak interactions," Nucl. Phys. B 373 (1992) 453.

[25] M. S. Carena, M. Quiros, M. Seco and C. E. M. Wagner, "Improved results in supersymmetric electroweak baryogenesis," Nucl. Phys. B 650 (2003) 24 arXiv:hep-ph/0208043.

[26] T. Konstandin, T. Prokopec, M. G. Schmidt and M. Seco, "MSSM electroweak baryogenesis and flavour mixing in transport equations," Nucl. Phys. B 738, 1 (2006) arXiv:hep-ph/0505103.

[27] V. Cirigliano, S. Profumo and M. J. Ramsey-Musolf, "Baryogenesis, electric dipole moments and dark matter in the MSSM," JHEP 0607, 002 (2006) arXiv:hep-ph/0603246.

[28] R. Barate et al. [LEP Working Group for Higgs boson searches and ALEPH Collaboration and and], "Search for the standard model Higgs boson at LEP," Phys. Lett. B 565 (2003) 61 arXiv:hep-ex/0306033.

S. Schael et al. [ALEPH Collaboration and DELPHI Collaboration and L3 Collaboration and ], "Search for neutral MSSM Higgs bosons at LEP," Eur. Phys. J. C 47 (2006) 547 arXiv:hep-ex/0602042.

[29] G. W. Anderson and L. J. Hall, "The Electroweak Phase Transition And Baryogenesis," Phys. Rev. D 45 (1992) 2685.

[30] S. J. Huber and T. Konstandin, "Production of Gravitational Waves in the nMSSM," JCAP 0805 (2008) 017 [hep-ph/0709.2091]. 
[31] A. Menon, D. E. Morrissey and C. E. M. Wagner, "Electroweak baryogenesis and dark matter in the nMSSM," Phys. Rev. D 70, 035005 (2004) arXiv:hep-ph/0404184].

[32] S. R. Coleman, "The Fate Of The False Vacuum. 1. Semiclassical Theory," Phys. Rev. D 15, 2929 (1977) [Erratum-ibid. D 16, 1248 (1977)].

[33] C. G. Callan and S. R. Coleman, "The Fate Of The False Vacuum. 2. First Quantum Corrections," Phys. Rev. D 16, 1762 (1977).

[34] A. D. Linde, "Fate Of The False Vacuum At Finite Temperature: Theory And Applications," Phys. Lett. B 100, 37 (1981);

"Decay Of The False Vacuum At Finite Temperature," Nucl. Phys. B 216 (1983) 421 [Erratum-ibid. B 223 (1983) 544].

[35] J. M. Moreno, M. Quiros and M. Seco, "Bubbles in the supersymmetric standard model," Nucl. Phys. B 526, 489 (1998) arXiv:hep-ph/9801272.

[36] T. Konstandin and S. J. Huber, "Numerical approach to multi dimensional phase transitions," JCAP 0606, 021 (2006) arXiv:hep-ph/0603081.

[37] J. M. Cline, G. D. Moore and G. Servant, "Was the electroweak phase transition preceded by a color broken phase?," Phys. Rev. D 60, 105035 (1999) arXiv:hep-ph/9902220.

[38] S. R. Coleman, "Quantum Tunneling And Negative Eigenvalues," Nucl. Phys. B 298 (1988) 178.

[39] A. Nicolis, "Relic gravitational waves from colliding bubbles and cosmic turbulence," Class. Quant. Grav. 21 (2004) L27 arXiv:gr-qc/0303084.

[40] C. Grojean and G. Servant, "Gravitational Waves from Phase Transitions at the Electroweak Scale and Beyond," Phys. Rev. D 75 (2007) 043507 arXiv:hep-ph/0607107.

[41] V. Corbin and N. J. Cornish, "Detecting the cosmic gravitational wave background with the big bang observer," Class. Quant. Grav. 23 (2006) 2435 arXiv:gr-qc/0512039.

[42] A. Kosowsky and M. S. Turner, Phys. Rev. D 47 (1993) 4372 arXiv:astro-ph/9211004.

[43] S. J. Huber and T. Konstandin, JCAP 0809 (2008) 022 [arXiv:0806.1828 [hep-ph]].

[44] A. D. Dolgov, D. Grasso and A. Nicolis, "Relic backgrounds of gravitational waves from cosmic turbulence," Phys. Rev. D 66 (2002) 103505 arXiv:astro-ph/0206461.

[45] C. Caprini and R. Durrer, "Gravitational waves from stochastic relativistic sources: Primordial turbulence and magnetic fields," Phys. Rev. D 74, 063521 (2006) arXiv:astro-ph/0603476. 